\title{
DRINFELD-SOKOLOV HIERARCHIES ON TRUNCATED CURRENT LIE ALGEBRAS
}

\author{
PAOLO CASATI \\ Dipartimento di matematica e applicazioni, Università di Milano-Bicocca \\ Via Cozzi 53, I-20125 Milano, Italy \\ E-mail:paolo.casati@unimib.it
}

\begin{abstract}
In this paper we construct on truncated current Lie algebras integrable hierarchies of partial differential equations, which generalize the Drinfeld-Sokolov hierarchies defined on Kac-Moody Lie algebras.
\end{abstract}

1. Introduction. Surely the discovery made by Drinfeld and Sokolov in their famous and celebrated paper [9], that to any affine Kac-Moody Lie algebra and to any vertex of its (extended) Dynkin diagram corresponds a hierarchy of completely integrable nonlinear partial differential equations, is a hallmark in the theory of integrable systems. These hierarchies (usually called Drinfeld-Sokolov hierarchies) have been further intensively studied in the last twenty years by many mathematicians with the help of various techniques (see [8, [1], 11, 4], 5], 15], 3], 27], 10], 16]).

The aim of this paper is to show how some generalizations of such hierarchies live on truncated current Lie algebras defined on affine Kac-Moody algebras. Roughly we shall proceed along the following lines. First we shall define, on the mentioned truncated current Lie algebras, Lax equations which are invariant with respect to a gauge action of a subgroup of the corresponding truncated current Lie groups. Then we shall show how such Lax equations give rise to integrable systems with an infinite number of conserved quantities.

Apart from the intrinsic interest of the generalizations considered here, the main output of such a construction is to obtain coupled integrable systems, which in recent years, have received considerable attention (see [6], [7, [17, [18], [19], 20], [21, [22, [23], [24], 28]).

2010 Mathematics Subject Classification: Primary 17B69, 37K10; Secondary 37K30.

Key words and phrases: Kac-Moody Lie algebras, partial differential equations.

The paper is in final form and no version of it will be published elsewhere. 
An outline of the paper is as follows. In the second section we briefly describe the truncated current Lie algebras and their properties used in this paper. In the third and last section we define on them evolution equations by means of Lax equations. Finally we show how they give rise to integrable systems endowed with a infinite number of conserved quantities.

2. Truncated current Lie algebras. In this section we wish to recall some facts on the truncated current Lie algebras. A fairly complete description of their properties may be found in [6] but see also [27]. The truncated current Lie algebras are quotients of loop algebras recently considered in the literature (sometimes under a different name), which may be defined explicitly as follows.

Let first denote by $\mathbb{C}[\xi]$ the commutative ring of all polynomials in the variable $\xi$ on the field $\mathbb{C}$ and let $\mathbb{C}^{(n)}$ be the its (finite dimensional) quotient ring $\mathbb{C}^{(n)}=\mathbb{C}[\xi] /\left(\xi^{n+1}\right)$, where $\left(\xi^{n+1}\right)$ is the principal ideal generated by $\xi^{n+1}$. Then we can give

Definition 2.1 ([27], 6] $)$. Let $\mathfrak{l}$ be a Lie algebra over the field $\mathbb{C}$. For any positive integer number $n \in \mathbb{N}$ the Lie algebra

$$
\mathfrak{l}^{(n)}=\mathfrak{l} \otimes \mathbb{C}^{(n)}
$$

is called a truncated current Lie algebra.

We can identify $\mathfrak{l}^{(n)}$ with the vector space of all polynomials in $\xi$ with coefficients in $\mathfrak{l}$ of a degree less than or equal to $n$ endowed with the Lie bracket

$$
\left[\sum_{j=0}^{n} X_{j} \xi^{j}, \sum_{i=0}^{n} Y_{i} \xi^{i}\right]=\sum_{k=0}^{n}\left(\sum_{j=0}^{k}\left[X_{j}, Y_{k-j}\right] \mathfrak{l}\right) \xi^{k} .
$$

where $[\cdot, \cdot]_{\mathfrak{l}}$ is the Lie bracket defined on $\mathfrak{l}$.

Observe that if $n>0$ then $\mathfrak{l}^{(n)}$ fails to be semisimple, because $\mathfrak{g} \otimes \xi^{n}$ is a non-trivial ideal. Moreover for $n>1$ the truncated Lie algebra $\mathfrak{l}^{(n)}$ may be regarded as a semidirect sum of $n$ Lie algebras "nested in each other". Nevertheless if $\mathfrak{l}$ admits a symmetric adinvariant non-degenerate bilinear form (i.e., if $\mathfrak{l}$ is a quadratic algebra) then roughly speaking this bilinear form is inherited by the whole Lie algebra $\mathfrak{l}^{(n)}$. Indeed we have:

THEOREM 2.2 ([6]). Suppose that a symmetric ad-invariant non-degenerate bilinear form $\langle\cdot, \cdot\rangle_{\mathfrak{l}}$ is defined on $\mathfrak{l}$. Then for any set of complex numbers $\mathcal{A}=\left\{a_{j}\right\}_{j=0, \ldots, n}$, the bilinear form

$$
\begin{array}{ll}
\mathfrak{l}^{(n)} \times \mathfrak{l}^{(n)} & \rightarrow \mathbb{C}, \\
(X(\xi), Y(\xi)) & \mapsto\langle X(\xi), Y(\xi)\rangle_{\mathcal{A}}^{(n)}=\sum_{j=0}^{n} a_{j} \sum_{i=0}^{j}\left\langle X_{i}, Y_{j-i}\right\rangle_{\mathfrak{l}},
\end{array}
$$

is a symmetric bilinear, ad-invariant and, if $a_{n} \neq 0$, non-degenerate form.

Further it is easy to construct a matrix realization of $\mathfrak{l}^{(n)}$ from one of $\mathfrak{l}$.

TheOREM $2.3([6])$. If $\Pi: \mathfrak{l} \rightarrow$ Aut $\left(\mathbb{C}^{m}\right)$ for some $m$ is a true representation of $\mathfrak{l}$ then the map

$$
\widetilde{\Pi}: \mathfrak{l}^{(n)} \mapsto \operatorname{Aut}\left(\mathbb{C}^{m(n+1)}\right)
$$


given by

$$
\widetilde{\Pi}\left(X_{0}, \ldots, X_{n}\right)=\left(\begin{array}{cccccc}
\Pi\left(X_{0}\right) & 0 & 0 & 0 & 0 & 0 \\
\Pi\left(X_{1}\right) & \Pi\left(X_{0}\right) & 0 & 0 & 0 & 0 \\
\vdots & \ddots & \ddots & \ddots & 0 & 0 \\
\vdots & \vdots & \ddots & \ddots & \ddots & \vdots \\
\Pi\left(X_{n-1}\right) & \vdots & \ldots & \cdots & \Pi\left(X_{0}\right) & 0 \\
\Pi\left(X_{n}\right) & \Pi\left(X_{n-1}\right) & \ldots & \cdots & \Pi\left(X_{1}\right) & \Pi\left(X_{0}\right)
\end{array}\right)
$$

is a faithful representation of $\mathfrak{l}^{(n)}$.

In what follows the Lie algebra $\mathfrak{l}$ is an affine nontwisted Kac-Moody Lie algebra $\widehat{\mathfrak{g}}$. We recall that an affine nontwisted Kac-Moody Lie algebra $\widehat{\mathfrak{g}}$ can be defined as follows.

Definition 2.4. The affine (non twisted) Lie algebra $\widehat{\mathfrak{g}}$ associated with the finite dimensional simple Lie algebra $\mathfrak{g}$ is the complex vector space

$$
\widehat{\mathfrak{g}}=\mathfrak{g} \otimes \mathbb{C}\left[z, z^{-1}\right] \oplus \mathbb{C} K \oplus \mathbb{C} d
$$

endowed with the following Lie bracket:

$$
\begin{aligned}
& {\left[P(z)+\mu_{1} K+\nu_{1} d, Q(z)+\mu_{2} K+\nu_{2} d\right]_{\widehat{\mathfrak{g}}}} \\
& \quad=[P(z), Q(z)]+\nu_{1} z \frac{d P(z)}{d z}-\nu_{2} z \frac{d Q(z)}{d z}+\left(\int_{S^{1}}\left\langle\frac{P(z)}{d z}, Q(z)\right\rangle_{\mathfrak{g}}\right) K
\end{aligned}
$$

where $[\cdot, \cdot]$ is the Lie bracket of the tensor Lie algebra $\mathfrak{g} \otimes \mathbb{C}\left[z, z^{-1}\right], K$ the central charge of the Kac-Moody Lie algebra $\widehat{\mathfrak{g}}$ and $d$ its derivation.

In what follows the derivation $d$ will not play any role.

Starting from the Killing form $\langle\cdot, \cdot\rangle_{\mathfrak{g}}$ of $\mathfrak{g}$ we can define an ad-invariant non-degenerate bilinear form $\langle\cdot, \cdot\rangle_{\widehat{g}}$ on $\widehat{\mathfrak{g}}[13$, and therefore a corresponding ad-invariant non-degenerate bilinear form $(\cdot, \cdot)$ on $\widehat{\mathfrak{g}}^{(n)}$ (see 2.2$)$ ).

In order to construct the Lax operator which we are interested in, we must first describe briefly the structure of a simple (finite dimensional) Lie algebra $\mathfrak{g}$.

Let $\mathfrak{h}$ be a Cartan subalgebra of $\mathfrak{g}$, and let $\Delta$ be the corresponding root system. Let $\Pi=\left\{\alpha_{1}, \ldots, \alpha_{r}\right\}$ be a set of simple roots in $\Delta$ (where $r$ is the rank of $\mathfrak{g}$ ), and $\Delta=\Delta^{+} \cup \Delta^{-}$the associated decomposition of $\Delta$ in positive (resp. negative) roots, then we have the Cartan decomposition

$$
\mathfrak{g}=\mathfrak{n}^{+} \oplus \mathfrak{h} \oplus \mathfrak{n}^{-}=\left(\bigoplus_{\alpha \in \Delta^{+}} \mathfrak{g}_{\alpha}\right) \oplus \mathfrak{h} \oplus\left(\bigoplus_{\alpha \in \Delta^{+}} \mathfrak{g}_{-\alpha}\right),
$$

where $\mathfrak{g}_{\alpha}=\{X \in \mathfrak{g} \mid[H, X]=\alpha(H) X \forall H \in \mathfrak{h}\}$ is the root space of $\alpha$. For any root $\alpha \in \Delta$, let $H_{\alpha}$ be the corresponding coroot with respect to the bilinear form $\langle\cdot, \cdot\rangle_{\mathfrak{g}}$, and for any $\alpha \in \Delta^{+}$let $X_{\alpha}$ be a basis of $\mathfrak{g}_{\alpha}$ and let $Y_{\alpha}$ in $\mathfrak{g}_{-\alpha}$ be defined by the requirement $\left[X_{\alpha}, Y_{\alpha}\right]=H_{\alpha}$. Let $\theta$ be the maximal root of $\Delta$ with respect to the above defined decomposition (i.e, $\theta+\alpha_{i} \notin \Delta$ for all $\alpha_{i} \in \Pi$ ). 
Let now $\Lambda$ be the element of $\widehat{\mathfrak{g}}$ defined by

$$
\Lambda=\sum_{i=1}^{r} X_{\alpha_{i}}+z Y_{\theta} .
$$

It can be shown (see [9], [11, 2] ) that $\Lambda$ is a regular element of $\widehat{\mathfrak{g}}$, i.e., that the centralizer $\Lambda$ is a regular semisimple element and therefore its isotropic subalgebra $\mathfrak{g}_{\Lambda}$ is a (Heisenberg) subalgebra $\mathfrak{H}$ of $\widetilde{\mathfrak{g}}$ spanned, in the case of the Kac-Moody Lie algebra of type $A_{n}^{(1)}, B_{n}^{(1)}$, $C_{n}^{(1)}$, by the matrices

$$
\begin{array}{llll}
\Lambda^{m} & m \in \mathbb{Z} & m \neq 0 \bmod (n+1) & \text { if } \mathfrak{g} \text { is of type } A_{n}, \\
\Lambda^{2 m+1} & m \in \mathbb{Z} & \text { where for } m<0, \Lambda^{2 m+1} \stackrel{\text { def }}{=} \lambda^{-k} \Lambda^{2 m+1+2 n k} & \text { if } \mathfrak{g} \text { is of type } B_{n}, \\
\Lambda^{2 m+1} & m \in \mathbb{Z} & \text { if } \mathfrak{g} \text { is of type } C_{n}
\end{array}
$$

(see [9]). In the more complicated case of the Lie algebra $D_{n}^{(1)}$ the Heisenberg Lie algebra $\mathfrak{H}$ is spanned by the matrices

$$
\Lambda^{2 m+1} \quad m \in \mathbb{Z} \quad \text { where for } m<0, \quad \Lambda^{2 m+1} \stackrel{\text { def }}{=} \lambda^{-k} \Lambda^{2 m+1+(2 n-2) k}
$$

together with the matrices

$$
\lambda^{m} F \quad m \in \mathbb{Z}, \quad F=\Phi+(-1)^{n} \Phi^{T} \quad \Phi=e_{n, 1}-2 e_{n+1,1}-2 e_{n, 2 n}+4 e_{n+1,2 n} .
$$

Here $e_{i j}$ is the matrix in $M(N, \mathbb{C})$ with 1 in the $i j$ position and zero anywhere else and $X^{T}$ denotes the transpose of $X$.

A similar result holds for the truncated current Lie algebra $\widehat{\mathfrak{g}}^{(n)}$. It is indeed easy to prove

Proposition 2.5. Let $\Lambda^{(n)} \in \widehat{\mathfrak{g}}^{(n)}$ be the element

$$
\Lambda^{(n)}=\sum_{i=0}^{n} \Lambda \xi^{i}
$$

then its isotropic subalgebra $\widehat{\mathfrak{g}}_{\Lambda^{(n)}}^{(n)}$ is a maximal abelian subalgebra of $\widehat{\mathfrak{g}}^{(n)}$ which coincides with $\mathfrak{H}^{(n)}=\oplus_{i=0}^{n} \mathfrak{H} \xi^{i}$.

A simple Lie algebra $\mathfrak{g}$ admits a decomposition w.r.t. the principal gradation (see [10], [8])

$$
\mathfrak{g}=\bigoplus_{1-h \leq j \leq h-1} \mathfrak{g}^{j}, \quad \mathfrak{g}^{j}= \begin{cases}\mathfrak{h} & \text { if } j=0, \\ \bigoplus_{h \mathrm{ht}(\alpha)=\mathrm{j}} \mathfrak{g}_{\alpha} & \text { if } j \neq 0,\end{cases}
$$

where ht is the height function of roots: if $\alpha=\sum_{\alpha_{i} \in \Pi} n_{i} \alpha_{i}$ then $h(t)=\sum_{i \in \Pi} n_{i}$ and $h$ is the Coxeter number of $\mathfrak{g}$. Such a gradation may be extended to the whole truncated current Lie algebra $\widehat{\mathfrak{g}}^{(n)}$ by setting $\operatorname{deg} z=h$ and $\operatorname{deg} \xi=0$. With respect to this gradation the subalgebra $\mathfrak{H}^{(n)}$ decomposes into homogeneous elements:

$$
\mathfrak{H}^{(n)}=\bigoplus_{i \in \mathbb{Z}} \mathfrak{H}_{i}^{(n)}
$$

and in particular $\Lambda^{(n)}$ is a homogeneous element of degree 1.

Finally let $\mathcal{L}(\widehat{\mathfrak{g}})$ be the loop algebra

$$
\mathcal{L}(\widehat{\mathfrak{g}})=C^{\infty}\left(S^{1}, \widehat{\mathfrak{g}}\right)
$$


and $\mathcal{L}(\widehat{\mathfrak{g}}) *$ be the space of linear functionals on $\widehat{\mathfrak{g}}$ of the following form:

$$
\mathcal{L}_{(P(x)+a K)}(Q(x)+b K)=\langle P(x)+a K, Q(x)+b K\rangle=\int_{S^{1}}\langle P(x), Q(x)\rangle_{\mathfrak{g}} d x+a b .
$$

We identify $\widehat{\mathfrak{g}}^{*}$ with $\mathcal{L}(\widehat{\mathfrak{g}})$. We further identify the tangent space at any point of $\widehat{\mathfrak{g}}$ with $\widehat{\mathfrak{g}}$ itself.

3. The Lax equations. We begin by defining a differential operator $L$, associated to the space $C^{\infty}\left(S^{1}, \widehat{\mathfrak{g}}\right)$, where $\widehat{\mathfrak{g}}$ is an untwisted affine Kac-Moody algebra.

Consider the Borel subalgebra of $\mathfrak{g}: \mathfrak{b}=\mathfrak{n}^{-} \oplus \mathfrak{h}$ and define

$$
\begin{aligned}
& \widehat{\mathfrak{n}}^{-}=\mathfrak{n}^{-} \otimes \mathbb{C}\left[z, z^{-1}\right], \quad \mathfrak{n}^{-(n)}=\mathfrak{n}^{-} \otimes \mathbb{C}^{(n)}, \quad \widehat{\mathfrak{n}}^{-(n)}=\widehat{\mathfrak{n}}^{-} \otimes \mathbb{C}^{(n)}, \\
& \widehat{\mathfrak{b}}=\mathfrak{b} \otimes \mathbb{C}\left[z, z^{-1}\right], \quad \mathfrak{b}^{(n)}=\mathfrak{b}^{-} \otimes \mathbb{C}^{(n)}, \quad \widehat{\mathfrak{b}}^{(n)}=\widehat{\mathfrak{b}}^{-} \otimes \mathbb{C}^{(n)} .
\end{aligned}
$$

Then the Lax operator $L$ is given by

$$
L=\partial_{x}+q+\Lambda^{(n)}
$$

where $\partial_{x}$ is the derivative along the variable $x \in S^{1}$ and $q \in C^{\infty}\left(S^{1}, \widehat{\mathfrak{b}}^{(n)}\right)$. The truncated current Kac-Moody group acts on $L([6])$. More precisely let us first give

Definition 3.1. Let $\widehat{G}$ be the Kac-Moody group of $\widehat{\mathfrak{g}}$ and define

$$
\widehat{G}^{(n)}=C^{\infty}\left(S^{1}, \widehat{G} \otimes \otimes \mathbb{C}^{(n)}\right) .
$$

Then there exists a formal action of the group $\widehat{G}^{(n)}$ on $L$, which we call a gauge transformation. The action is defined by the exponentiated adjoint action:

$$
L_{0}=\exp (\operatorname{adT})(L), \quad T \in C^{\infty}\left(S^{1}, \widehat{\mathfrak{g}}^{(n)}\right),
$$

where the exponentiated adjoint action is defined by its power series expansion, i.e.

$$
\exp (\operatorname{adT})(a)=a+[T, a]+\frac{1}{2}[T,[T, a]]+\frac{1}{3 !}[T,[T,[T, a]]]+\cdots
$$

Observe that, if $L$ is an operator of the form 3.1 and $T \in C^{\infty}\left(S^{1}, \widehat{\mathfrak{n}}^{(n)}\right)$, then the operator

$$
\widetilde{L}=\exp (\operatorname{adT})(L)
$$

also has the form (3.1), because

$$
\left[\widehat{\mathfrak{n}}^{(n)}, \widehat{\mathfrak{b}}^{(n)}\right] \subset \widehat{\mathfrak{n}}^{(n)}, \quad\left[\widehat{\mathfrak{n}}^{(n)}, Y_{\theta} \otimes \xi^{k}\right]=0, \quad\left[\widehat{\mathfrak{n}}^{(n)}, \sum_{i=1}^{r} X_{\alpha_{i}} \otimes \xi^{k}\right] \subset \widehat{\mathfrak{b}}^{(n)} \quad 0 \leq k \leq n .
$$

Let $\widehat{\mathfrak{b}}_{0}^{(n)}=\widehat{\mathfrak{h}}^{(n)}=\mathfrak{h} \otimes \mathbb{C}^{(n)}$ and $\widehat{\mathfrak{b}}_{i}^{(n)}=\mathfrak{g}^{i} \otimes \mathbb{C}\left[z, z^{-1}\right] \otimes \mathbb{C}^{(n)}(0 \leq i \leq h-1)$. Then it follows from [9] (proposition 3.3 page 1990) and the definition of truncated current Lie algebra that for any $i>0$ the operator $\operatorname{ad}\left(\sum_{i=1}^{r} X_{\alpha_{i}}\right)$ acts from $\widehat{\mathfrak{b}}_{i}^{(n)}$ to $\widehat{\mathfrak{b}}_{i-i}^{(n)}$ injectively. Let $J$ be the set of all $i, 1 \leq h-1$, such that the kernel $V_{i}^{(n)}$ of $\operatorname{ad}\left(\sum_{i=1}^{r} X_{\alpha_{i}}\right): \widehat{\mathfrak{b}}_{i}^{(n)} \rightarrow \widehat{\mathfrak{b}}_{i-1}^{(n)}$ is non-trivial and set $V^{(n)}=\oplus_{i \in J} V_{i}^{(n)}$.

Proposition 3.2. Any operator $L$ of the form 3.1 can be uniquely represented in the form $L=\exp (a d S)(\widetilde{L})$, where $S \in C^{\infty}\left(S^{1}, \widehat{\mathfrak{n}}^{(n)}\right)$ and

$$
\widetilde{L}=L=\partial_{x}+\widetilde{q}+\Lambda^{(n)}, \quad \widetilde{q} \in C^{\infty}\left(S^{1}, V^{(n)}\right) .
$$


Proof. Since $\left[S, Y_{\theta}\right]=0$, the proof boils down to showing that we can represent $\partial_{x}+q+\sum_{k=0}^{n}\left(\sum_{i=1}^{r} X_{\alpha_{i}}\right) \xi^{k}$ in the form $\exp (\operatorname{ad}(S))\left(\partial_{x}+\widetilde{q}+\sum_{k=0}^{n}\left(\sum_{i=1}^{r} X_{\alpha_{i}}\right) \xi^{k}\right)$. Let $\widetilde{q}=\sum_{i=0}^{h-1} \widetilde{q}_{i}, S=\sum_{i=0}^{h-1} S_{i}$, where $\widetilde{q}_{i}, S_{i} \in C^{\infty}\left(S^{1}, \widehat{\mathfrak{b}}_{i}^{(n)}\right)$ with $\widetilde{q}_{i}=\sum_{k=0}^{n} \widetilde{q}_{i, k} \xi^{k}$ and $S_{i}=\sum_{k=0}^{n} S_{i, k} \xi^{k}$. Equating components lying in $C^{\infty}\left(S^{1}, \widehat{\mathfrak{b}}_{i}^{(n)}\right)$ in the expression $\partial_{x}+q+$ $\left.\sum_{k=0}^{n}\left(\sum_{i=1}^{r} X_{\alpha_{i}}\right) \xi^{k}=\exp (\operatorname{ad}(S))\left(\partial_{x}+\widetilde{q}+\sum_{k=0}^{n}\left(\sum_{i=1}^{r} X_{\alpha_{i}}\right)\right) \xi^{k}\right)$, and considering the elements with the same power in $\xi$ we find that $\widetilde{q}_{i, k}+\sum_{j=0}^{k}\left[S_{i+1, j}, \sum_{k=0}^{n}\left(\sum_{i=1}^{r} X_{\alpha_{i}}\right)\right]$, $0 \leq k \leq n$, can be expressed in terms of $\widetilde{q}_{0, l} \ldots, \widetilde{q}_{i-1, l}$ and $S_{1, l} \ldots, S_{i, l} 0 \leq l \leq k$. Therefore, if $\widetilde{q}_{0, l} \ldots, \widetilde{q}_{i-1, l}$ and $S_{1, l} \ldots, S_{i, l}, 0 \leq l \leq k$, have already been found, then $\widetilde{q}_{i, k}$ and $S_{i+1, k}$ can be uniquely determined because $\operatorname{ad}\left(\sum_{i=1}^{r} X_{\alpha_{i}}\right)$ is injective.

We can now write down the equations for the class of gauge equivalence which are coupled soliton equations.

THEOREM 3.3. There exists a (non-unique) gauge transformation of the form given above, where $T \in C^{\infty}\left(S^{1}, \oplus_{i<0} \widehat{\mathfrak{g}}_{i}^{(n)}\right)$ (where $\widehat{\mathfrak{g}}_{i}^{(n)}$ is the subspace spanned by the element of degree $i$ with respect to the principal gradation), such that $L_{0}$ has the form

$$
L_{0}=\partial_{x}+\Lambda+\sum_{i<1} H_{i}
$$

with $H_{i} \in C^{\infty}\left(S^{1}, \mathfrak{H}_{i}\right)$

Proof. If we equate the terms of the same grade with respect to the principal gradation in the expression $\exp (\operatorname{adT})(L)=L_{0}$ we obtain a recursion relation of the form

$$
\sum_{k=0}^{n} H_{i, k} \xi^{k}+\sum_{k=0}^{n}\left(\sum_{j=0}^{k}\left[\Lambda, T_{i-1, k-j}\right]_{\mathfrak{g}}\right) \xi^{k}=\sum_{k=0}^{n} P_{k} \xi^{k}
$$

where $T_{j}$ is the component of $\mathrm{T}$ of grade $j$, and the elements $P_{k}$ are terms of grade $j$ which depend on $H_{l}$, for $l>j, T_{l}$, for $l>j-1$, and $q$. This latter equation implies

$$
H_{i, k}+\sum_{j=0}^{k}\left[\Lambda, T_{i-1, k-j}\right]=P_{k} \quad k=0, \ldots, n .
$$

Now we can proceed by induction: the first set of equations of the series are

$$
H_{0, k}+\sum_{j=0}^{k}\left[\Lambda, T_{-1, k-j}\right]=q_{0, k}
$$

where $q_{0, k}$ is the component of $q_{k}$ of degree 0 . The equation for $k=0$ reads

$$
H_{0,0}+\left[\Lambda, T_{-1,0}\right]=q_{0,0},
$$

which can be regarded as an equation in $\widehat{\mathfrak{g}}$. It is well known ([9] and [11]) that $\Lambda$ is a regular element, $\widehat{\mathfrak{g}}$ has the decomposition $\widehat{\mathfrak{g}}=\operatorname{Ker}(\operatorname{ad}(\Lambda)) \oplus \operatorname{Im}(\operatorname{ad}(\Lambda))$ with $\operatorname{Ker}(\operatorname{ad}(\Lambda))=\mathfrak{H}$. Hence, we can solve it uniquely for $H_{0,0}$, and for $T_{-l, 0}$ up to an element of $\operatorname{Ker}(\operatorname{ad}(\Lambda))$. Now for $k=1$ equation 3.6 becomes

$$
H_{0,1}+\left[\Lambda, T_{-1,0}\right]+\left[\Lambda, T_{-1,1}\right]=q_{0,1},
$$

which again can be solved uniquely for $H_{0,1}$, and for $T_{-l, 1}$ up to an element of $\operatorname{Ker}(\operatorname{ad}(\Lambda))$. It is clear that iteratively we can solve (uniquely for $H_{0, k}$, and for $T_{-l, k}$ up to an element 
of $\operatorname{Ker}(\operatorname{ad}(\Lambda)))$ any equation in (3.6). The same argument shows that we can solve any equation in 3.5 iteratively. Observe that we can fix the freedom in the $T_{j, k}$ (i.e., that if $T_{j, k}$ solves an equation in 3.6 then also $T_{j, k}+\chi$ with $\chi \in \operatorname{Ker}(\operatorname{ad}(\Lambda))$ solves the same equation) by demanding that $T_{j, k} \in \operatorname{Im}(\operatorname{ad}(\Lambda))$.

For any $L$ of type 3.1 define $Z_{L}=\left\{M \in C^{\infty}\left(S^{1}, \widehat{G}^{(n)}\right) \mid[M, L]=0\right\}$.

Corollary 3.4. $Z_{L}=\exp (\operatorname{adT})(L)$, where $T$ is as in Theorem 3.3.

Proof. We only have to prove that $Z_{L_{0}}=\mathfrak{H}$. Let $\left[M, L_{0}\right]=0, M=\sum_{i=-\infty}^{n} M_{i}, M_{i} \in \widehat{\mathfrak{g}}_{i}^{(n)}$. Equating to zero the component of $\widehat{\mathfrak{g}}_{n+1}^{(n)}$, we find that $M_{n} \in C^{\infty}\left(S^{1}, \mathfrak{H}\right)$. Equating now to zero the component of $\widehat{\mathfrak{g}}_{n}^{(n)} \mathrm{G}$, we obtain $\partial M_{n}+\left[M_{n-1}, L_{0}\right]$. Since the left side of this equality belongs to $C^{\infty}\left(S^{1}, \mathfrak{H}\right)$, while the right side lies in $C^{\infty}\left(S^{1}, \operatorname{Im}(\operatorname{ad}(\Lambda))\right.$ and these two spaces have trivial intersection as a consequence of the properties of $\Lambda$ (see previous section), we have $\partial_{x} M_{n}=0$, i.e., $M_{n} \in \mathfrak{H}$. We then apply analogous considerations to $M-M_{n}$

Lemma 3.5. Let $M \in Z_{L}$ and denote by $M_{+}$(resp. $\left.M_{-}\right)$its canonical projections on $C^{\infty}$ $\left(S^{1}, \oplus_{i \geq 0} \widehat{\mathfrak{g}}_{i}^{(n)}\right)\left(\right.$ resp. on $\left.C^{\infty}\left(S^{1}, \oplus_{i \leq 0} \widehat{\mathfrak{g}}_{i}^{(n)}\right)\right)$ then $\left[L, M_{+}\right]$belongs to $C^{\infty}\left(S^{1}, \mathfrak{b}^{(n)}\right)$.

Proof. Observe first that with our definition any positive element (resp. any strictly negative element) in the principal gradation is a sum of elements which are non negative (resp. which are non strictly positive) powers in $z$. From $[M, L]=0$, we have $\left[M_{+}, L\right]=$ $-\left[M_{-}, L\right]$. The right side of this equality does not contain components with positive power in $z$, because $L$ is the sum of $z Y_{\theta}$ and of an element which does not contain positive powers in $z$, similarly $M_{-}$can be written as a sum of elements in $\mathfrak{n}^{(n)}$ and elements which do not contain positive powers in $z$, but $z Y_{\theta}$ commutes with $\mathfrak{n}^{(n)}$. Therefore in the principal gradation the right side is nonpositive, while the left is nonnegative.

Using Proposition 3.2. Theorem 3.3 and Lemma 3.5 , we prove the

Proposition 3.6. There exist an infinite number of commuting flows of the form

$$
\frac{d L}{d t_{u}}=\left[\exp (a d(T) u)_{+}, L\right]
$$

$u$ is an element of $\mathfrak{H}^{(n)}$.

Proof. Only the commutativity of the flows remains to be proved, which follows from the same argument of [9] sec 3 and 6 and [11] proposition 3.1.

The following proposition follows also from the same references.

Proposition 3.7. The quantities $H_{j}, j \leq 0$, of (3.4) are the conserved densities for the hierarchy.

4. Conclusion. In this paper we have shown how the Drinfeld-Sokolov construction of hierarchies of soliton equations can be extended from the Kac-Moody Lie algebras to the truncated Kac-Moody Lie algebras. In this way we obtain hierarchies of coupled soliton equations of the type recently studied in [18, [19, [20], [21, [22, [23], 24]. Such approach allows also to construct a wide set of soliton solutions. 
Acknowledgments. The author wishes to thank Professor Zbigniew Hajto for his patience and kindness, and the anonymous referee for the many welcomed suggestions which have improved this paper considerably.

\section{References}

[1] N. J. Burroughs, M. F. de Groot, T. Hollowood and J. L. Miramontes, Generalized Drinfel'd-Sokolov hierarchies. II. The Hamiltonian structures, Comm. Math. Phys. 153 (1993), 187-215.

[2] P. Casati, A new construction of the Drinfeld-Sokolov hierarchies, submitted for publication in Communication on Mathematical Physics.

[3] P. Casati, A. Della Vedova and G. Ortenzi, The soliton equations associated with the affine Kac-Moody Lie algebra $G_{2}^{(1)}$, J. Geom. Phys. 58 (2008), 377-386.

[4] P. Casati, G. Falqui, F. Magri and M. Pedroni, Bi-Hamiltonian reductions and $w_{n}$ algebras, J. Geom. Phys. 26 (1998), 291-310.

[5] P. Casati, G. Falqui, F. Magri and M. Pedroni, A note on fractional KdV hierarchies, J. Math. Phys. 38 (1997), 4606-4628.

[6] P. Casati and G. Ortenzi, New integrable hierarchies from vertex operator representations of polynomial Lie algebras, Journal of Geometry and Physics 56 (2006), 418-449.

[7] P. Casati and G. Ortenzi, Bihamiltonian equations on polynomial Virasoro algebras, Journal of Nonlinear Mathematical Physics 13 (2006), 352-364.

[8] P. Casati and M. Pedroni, Drinfeld-Sokolov reduction on a simple Lie algebra from the bi-Hamiltonian point of view, Lett. Math. Phys. 25 (1992), 89-101.

[9] V. G. Drinfeld and V. V. Sokolov, Lie algebras and equations of Korteweg-de Vries type, J. Sov. Math. 30 (1985), 1975-2036.

[10] B. Dubrovin, S. Liu and Y. Zhang, Frobenius manifolds and central invariants for the Drinfeld-Sokolov bihamiltonian structures, Advances in Mathematics 219 (2008), 780-837.

[11] M. F. de Groot, T. Hollowood and J. L. Miramontes, Generalized Drinfel'd-Sokolov hierarchies, Comm. Math. Phys. 145 (1992), 57-84.

[12] R. Hirota, X. Hu and X. Tang, A vector potential KdV equation and vector Ito equation: soliton solutions, bilinear Bäcklund transformations and Lax pairs, J. Math. Anal. Appl. 288 (2003), 326-348.

[13] V. G. Kac, Infinite Dimensional Lie Algebras, 3rd ed., Cambridge University Press, Cambridge, 1990.

[14] S. Kakei, Dressing method and the coupled KP hierarchy, Phys. Lett. A 264 (2000), 449458.

[15] B. Khesin and F. Malikov, Universal Drinfel'd-Sokolov reduction and matrices of complex size, Comm. Math. Phys. 175 (1996), 113-134.

[16] S. Liu, C. Wu and Y. Zhang, On the Drinfeld-Sokolov hierarchies of D type, International Mathematics Research Notices 2010, Article ID rnq138, 45 pp.

[17] W. X. Ma, Integrable couplings of vector AKNS soliton equations, J. Math. Phys. 46 (2005), 033507, 19 pp.

[18] W. X. Ma, Integrable couplings of soliton equations by perturbations I. A general theory and application to the KdV hierarchy, Methods Appl. Anal. 7 (2000), 21-56.

[19] W. X. Ma and M. Chen, Hamiltonian and quasi-Hamiltonian structures associated with semi-direct sums of Lie algebras, J. Phys. A: Math. Gen. 39 (2006), 10787-10801. 
[20] W. X. Ma and E. G. Fan, Linear superposition principle applying to Hirota bilinear equations, Computers and Mathematics with Applications 61 (2011), 950-959.

[21] W. X. Ma and B. Fuchssteiner, Integrable theory of the perturbation equations, Chaos Solitons Fract. 7 (1996), 1227-1250.

[22] W. X. Ma and L. Gao, Coupling integrable couplings, Modern Phys. Lett. B 23 (2009), $1847-1860$.

[23] W. X. Ma, X. X. Xu and Y. F. Zgang, Semi-direct sums of Lie algebras and continuous integrable couplings, Phys. Lett. A 351 (2006), 125-130.

[24] W. X. Ma and Z. N. Zhu, Constructing nonlinear discrete integrable Hamiltonian couplings, Computers and Mathematics with Applications 60 (2010), 2601-2608.

[25] A. Medina and P. Revoy, Algèbres de Lie et produit scalaire invariant, Ann. Sci. École Norm. Sup. (4) 18 (1985), 553-561.

[26] S. Yu. Sakovich, A note in the Painlevé property of coupled KdV equation, arXiv:nlin.SI/ 0402004.

[27] C. Z. Wu, A remark on Kac-Wakimoto hierarchies of D-type, Journal of Physics A: Mathematical and Theoretical 43 (2010), 035201.

[28] X. X. Xu, An integrable coupling hierarchy of the Mkdv integrable systems, its Hamiltonian structure and corresponding nonisospectral integrable hierarchy, Applied Mathematics and Computation 216 (2010), 344-353. 
